\title{
The psychometric properties of the Barkin index of maternal functioning (BIMF) for the Iranian population
}

\author{
Mojgan Mirghafourvand', Jennifer L. Barkin², Mohammad Asghari Jafarabadi ${ }^{3,4}$, Fatemeh Karami ${ }^{5}$ and \\ Solmaz Ghanbari-Homayi ${ }^{6^{*}}$
}

\begin{abstract}
Background: Mothers' capability for childcare and compatibility with the maternal role represent important challenges in postpartum care. Given the significance of evaluating maternal functioning, and the lack of adequate standard instruments in Iran for this purpose, the present study was aimed at translating and conducting a psychometric assessment of the Barkin Index of Maternal Functioning (BIMF) for Iranian women.

Methods: The instrument was translated into Persian using the Backward Forward method. The study included 530 women in the postpartum period admitted to healthcare centers in Tabriz, Iran; they were selected through the cluster sampling method. Face, content, and construct (through exploratory and confirmatory analyses) validity were presently examined. Reliability of the questionnaire was determined using the internal consistency and testretest reliability methods.

Results: Two factors (mom's needs and competency), emerged based on exploratory factor analysis. The $x^{2} / d f$ ratio was less than 5, and the values of the Root Mean Square Error of Approximation (RMSEA) and the Root Mean Square Residual (RMR) were less than 0.08 and 0.1 , respectively, verifying the model validity. Cronbach's alpha coefficient and Intra-class Correlation Coefficient (ICC) were calculated as 0.88 and 0.85 , respectively, indicating reliability.
\end{abstract}

Conclusion: The Persian version of the BIMF is a valid and reliable instrument for measuring the postpartum functioning of Iranian mothers.

Keywords: Motherhood function, Postpartum period, Validity, Reliability, Psychometric, Iran

\section{Background}

Childbirth is a significant and life-altering experience for women who choose to have children [1]. In the postpartum period, women face significant changes in their cognitive, behavioral, and social functions $[2,3]$ and are required to adapt and integrate additional responsibilities into their existing set of roles and responsibilities [4]. This process can be both enjoyable and extremely challenging [5].

Postpartum maternal functioning encompasses various dimensions, including personal care, child and family

\footnotetext{
* Correspondence: homayisolmaz@gmail.com

${ }^{6}$ Department of Midwifery, Faculty of Nursing and Midwifery, Tabriz University of Medical Sciences, Tabriz, Iran

Full list of author information is available at the end of the article
}

care, and social and occupational activities [6]. Maternal functioning is reportedly improved substantially between the first and sixth postpartum weeks [7]; however, a number of women take up to six months to achieve a desirable level of functioning [8].

Accordingly, levels of maternal functioning vary among mothers, with numerous contributing factors such as maternal age, education, parity, unintended pregnancy, type of delivery [9], postpartum maternal mental health status [9], perceived support [10], and the prevailing sociocultural perspectives in society [11]. Optimal maternal functioning contributes positively to neonatal development and maternal-neonatal bonding, whereas suboptimal functioning may operate in an opposite fashion $[11,12]$.

(c) The Author(s). 2019 Open Access This article is distributed under the terms of the Creative Commons Attribution 4.0 International License (http://creativecommons.org/licenses/by/4.0/), which permits unrestricted use, distribution, and reproduction in any medium, provided you give appropriate credit to the original author(s) and the source, provide a link to the Creative Commons license, and indicate if changes were made. The Creative Commons Public Domain Dedication waiver (http://creativecommons.org/publicdomain/zero/1.0/) applies to the data made available in this article, unless otherwise stated. 
Proper assessment of postpartum maternal functioning is key to identifying mothers who might be struggling in the maternal role and require extra support in order to adapt optimally. In a recent study conducted in the United States, BIMF scores were 16 points higher $(p<$ $0.0001)$ on average, after women participated in a community-based non-clinical intervention [13]. The intervention consisted of trained volunteers providing social support to new mothers who either requested it or were referred. This results of this study point to the powerful effect of social support in the postpartum period.

Until recently, the Inventory of Functional Status After Childbirth (IFSAC) was the only instrument developed to measure postpartum maternal functioning. However, subsequent reports have pointed to potential dubious research results in studies using unmodified versions of this instrument. This is because the IFSAC was developed based on the assumption that optimal postpartum maternal functioning is contingent upon mothers resuming the majority of their prepartum responsibilities [14-16]. However, prepartum and postpartum functioning are not comparable, as childbirth often requires a reevaluation of one's activities. In fact, due to the IFSAC's scoring algorithm and inherent penalty for reprioritization after childbirth, the majority of new mothers found it difficult to return to their previous functional status $[16,17]$. Additionally, maternal psychological well-being, women's understanding of their new role, and their thoughts and feelings about adjustment over the first postpartum year, are not factored into the IFSAC's assessment of functioning [6].

The BIMF was developed as a multidimensional instrument for measuring maternal functioning in both clinical and research settings. It was designed in 2010 by Barkin et al. based on the views of 31 postpartum mothers, which were expressed in focus group discussions. It has been argued that, in addition to accessing information about the meanings of desirable and undesirable conditions from individual perspectives, this method makes it possible to get acquainted with the language of the target population [18]. This instrument consists of 20 items that cover all aspects of maternal functioning, including self-care [11], infant care, motherchild interaction, family management, psychological well-being, adjustment, and social support [6, 18-20].

Given the importance of evaluating maternal functioning and the lack of adequate valid instruments in Iran for this purpose, the present study was aimed at translating the tool and conducting a psychometric assessment of the BIMF for Iranian women. The aim of the present study was to adapt the BIMF to the Iranian culture and determine its psychometric properties.

\section{Methods}

\section{Study's participants}

Those eligible to participate were primiparous women with a vaginal delivery, single pregnancy, self-declared physical health, absence of stressful incidents such as divorce, death of next of kin, and diagnosis of a terminal or refractory diseases for a family member in the past three months, and a lack of a history of depression or any other mental disorder during the pre-pregnancy, prepartum, and postpartum periods (as declared by the participant). Mothers of infants who were hospitalized or had major neonatal anomalies were excluded.

\section{Sample size}

In this study, the sample size required to determine the construct validity of the instrument was considered 10 participants per item [21]. Given the number of questionnaire items $(n=20)$, a sample size of 200 was required. However, the total desired sample size was increased to 530, considering the cluster sampling method (taking into account the design effect equal with $2.5)$ and an attrition rate of $5 \%$.

\section{Measurement of functioning: the Barkin index of maternal functioning}

The BIMF was used to assess maternal functioning. The BIMF is of comprised 20 items with response options on a 6-point Likert scale. Maternal needs (questions 2, 6, 7, $8,9,11$, and 13) and maternal competency (questions 1 , $3,4,5,10,12,14,15,16,17,18,19$, and 20 ) were the two underlying factors which emerged in a psychometric evaluation by Barkin et al. [6]. In order to complete the BIMF, Mothers are asked to select the response that best reflects their experience over the past two weeks. The overall maternal functioning score (after reverse-scoring Items 16 and 18) ranges from 0 to 120 , with higher scores representing better functioning. In the original study, the Cronbach's alpha for the BIMF was reported as acceptable $(\alpha=0.87)[6,18]$.

\section{Translation process}

Written permission was first obtained from the instrument developer (Dr. Jennifer L. Barkin) for adaptation with the Iranian culture. The original version of the instrument was translated from English into Persian by a native English speaker who was also fluent in Persian. The translated version was reviewed by the research team. The previous version was then translated from Persian into English. At this stage, the translation was done by two translators fluent in both languages who were not involved in the previous stage. The translated version was subsequently reviewed by two individuals fluent in both languages and familiar with the terminology, who prepared the final version. 


\section{Sampling method}

Sampling was performed using the cluster method. About one-third of all healthcare centers in Tabriz, Iran, were first selected randomly using the website, www.random.org. Subsequently, the list of mothers during their sixth to tenth postpartum week was prepared based on the medical records at each center. Eligible individuals were randomly selected from the list and invited to participate in the research. The study objectives were then fully explained to mothers via in-person meetings, and the questionnaires were filled out by the participants. It should be noted that mothers were given necessary explanation regarding the study and information regarding confidentiality. Informed written consents were also obtained.

\section{Data collection}

Data collection instruments consisted of a sociodemographic questionnaire and the BIMF, which were completed by the participants from the sixth postpartum week up to the tenth. The socio-demographic questionnaire employed in this study included items regarding maternal age, education, occupation, income status, and unwanted pregnancy. The question about income has been designed as qualitative and the participants' response was based on their perception of sufficiency of income for household expenses.

\section{Face and content validity of the Barkin index of maternal functioning}

In order to determine the face validity, eight experts in midwifery, reproductive health, and psychiatric nursing were asked to evaluate all items in terms of simplicity and transparency. Based on the answers, the impact of each item was then calculated using the following formula from the score 1 (completely difficult or nontransparent) to 4 (completely simple and transparent) on the Likert scale [Impact $=$ significance (the mean value of the answers to the item) * frequency (number of score 4 $\mathrm{s}$ selected)]. Values less than 1.5 resulted in the removal of the item [22].

Content validity was determined quantitatively and qualitatively. For the qualitative portion, the experts who participated in the assessment of face validity were asked to review the translation of each item in terms of grammar, use of proper vocabulary, and proper placement of phrases, and offer their critical comments and revisions. Content validity index (CVI) and content validity ratio (CVR) were used for the quantitative method. CVI was determined by assessing items in terms of relevance, transparency, and simplicity based on a 4-point Likert scale. Scores greater than 0.79 were considered acceptable. In order to determine CVR, experts were asked to examine each item in terms of necessity based on a 4- point Likert scale. The minimum acceptable CVR was considered higher than $0.62[23,24]$.

\section{Construct validity}

Construct validity was assessed through exploratory and confirmatory factor analyses. Exploratory factor analysis was conducted using the Kaiser-Meyer-Olkin (KMO) measure of sampling adequacy and oblimin rotation. Factors were extracted using principal component analysis with varimax rotation, and the number of factors was determined using a scree plot of eigenvalues. An eigenvalue is the amount that determines the variance explained by a factor throughout a dataset. Therefore, the greater the eigenvalue of a factor, the greater its explanatory power of the variance.

The factor analysis method examines the internal relationship between variables and is used to extract categories of items that are most strongly related to each other. In this analysis, items with factor loadings of less than 0.3 were considered candidates for removal. Items with factor loadings of $0.3-0.5$ were kept in or removed from the instrument at the research team's discretion. After extracting factors and expressions therein, their consistency with the dimensions of the original questionnaire was examined [25].

In order to assess the structure of extracted factors from exploratory factor analysis, the model was evaluated using confirmatory factor analysis. Goodness of Fit Index (GFI) was used to assess the exploratory model fit. In order to verify the model, the following indices were determined as follows: Root Mean Square Error of Approximation (RMSEA) $<0.08$, the Standardized Root Mean Square Error of Approximation (SRMSEA) $<0.08$, Comparative Fit Index (CFI) $\geq 0.90$, Tucker-Lewis Index $(\mathrm{TLI}) \geq 0.95$, and normed chi-square $\left(\mathrm{x}^{2} / \mathrm{df}\right)<5.0[25$, 26].

\section{Discriminant validity}

The discriminant validity and differences between the known maternal age and spousal/family/friend support groups was determined using the known-groups method and Pearson's correlation coefficient. Studies suggest that younger mothers who receive support enjoy a better maternal functioning $[6,10]$.

\section{Reliability}

Reliability of the questionnaire was determined using the internal consistency and test-retest reliability methods. Internal consistency and test-retest reliability were examined by calculating the Cronbach's alpha and the ICC, respectively. Test-retest reliability was evaluated among the mothers who completed the questionnaire twice within a period of two weeks. 


\section{Results}

\section{Participants' profile}

A total of 530 mothers entered the study between August and January of 2018. The mean participant age was 27 years old; the majority of the participating mothers (95.1\%) were stay-at- home mothers. Other characteristics are given in Table 1.

\section{Face and content validity}

The study of face validity indicated that all items had been described as simple and transparent and received a minimum score of 1.5 . The study of content validity revealed that all items achieved the minimum acceptable values of CVI and CVR (Table 2).

\section{Construct validity}

Exploratory factor analysis was performed on 20 items through the principal component analysis method. The KMO value was calculated as 0.872. Bartlett's test achieved a value of 5853.49 at a significant level of less than 0.001 , justifying the implementation of factor analysis on the sample based on the correlation matrix.

The number of factors was determined using a scree plot of eigenvalues. Results demonstrated that the highest percentage of the total variance (44.2\%) was explained by the first two factors. The remaining of the total variance $(12.1 \%)$ was explained by the three succeeding factors. Accordingly, two factors with high eigenvalues were identified by this method that accounted for $44.2 \%$ of the total variance. Using the scree plot method, two factors were located on the first descending slope. Therefore, using this method, two factors were confirmed. In this study, Item 15 with a factor loading of

Table 1 Characteristics of the study participants $(n=530)$

\begin{tabular}{ll}
\hline Characteristics & $\mathrm{N}(\%)$ \\
\hline Age (years) & $27.0(5.4)$ \\
Education & $231(43.5)$ \\
$\quad$ High school or below & $195(36.8)$ \\
Diploma & $104(19.6)$ \\
College & \\
Job & $504(95.1)$ \\
Housewife & $26(4.9)$ \\
Employee & \\
Income & $80(15.1)$ \\
$\quad$ Not at all sufficient & $393(74.2)$ \\
Relatively sufficient & $57(10.8)$ \\
Completely sufficient & $20(3.8)$ \\
Unwanted sex of baby & $127(24.0)$ \\
Unwanted pregnancy & $38.5(2.1)$ \\
Gestational age at birth (weeks)
\end{tabular}

Table 2 The impact score, CVI, and CVR for Barkin Index of Maternal Functioning (BIMF)

\begin{tabular}{|c|c|c|c|}
\hline BMFI & Impact score & $\mathrm{CVl}^{\mathrm{a}}$ & $C V R^{b}$ \\
\hline & $n=8$ Experts & & \\
\hline BMFI1 & 3.04 & 0.87 & 0.87 \\
\hline BMFI2 & 4 & 1 & 1 \\
\hline BMFI3 & 4 & 1 & 1 \\
\hline BMFI4 & 3.04 & 0.79 & 0.87 \\
\hline BMFI5 & 4 & 1 & 1 \\
\hline BMFI6 & 4 & 1 & 1 \\
\hline BMFI7 & 4 & 1 & 1 \\
\hline BMFI8 & 3.04 & 0.91 & 1 \\
\hline BMFI9 & 4 & 1 & 1 \\
\hline BMFI10 & 4 & 0.91 & 0.87 \\
\hline BMFI11 & 4 & 0.91 & 1 \\
\hline BMFI12 & 4 & 1 & 1 \\
\hline BMFI13 & 4 & 1 & 1 \\
\hline BMFI14 & 4 & 1 & 1 \\
\hline BMFI15 & 4 & 0.79 & 0.75 \\
\hline BMFI16 & 4 & 1 & 1 \\
\hline BMFI17 & 4 & 1 & 1 \\
\hline BMFI18 & 3.04 & 0.95 & 1 \\
\hline BMFI19 & 4 & 1 & 1 \\
\hline BMFI20 & 4 & 1 & 1 \\
\hline
\end{tabular}

${ }^{\mathrm{a} C V I}$ Content Validity Index ${ }^{\mathrm{b}} \mathrm{CVR}$ Content Validity Ratio

less than 0.3 and Item 18 with a factor loading of $0.3-$ 0.5 were removed. In addition, Item 16 was not included under any of the factors, hence removed from the Persian version. Finally, the Persian version of the questionnaire was verified with 17 items and two factors, i.e. maternal needs (items 6, 7, and 8) and maternal competency (items 1, 2, 3, 4, 5, 9, 10, 11, 12, 13, 14, 17, 19, and 20) (Table 3).

Given the values of indices in Table 4 , the $x^{2} / d f$ ratio was smaller than 5, and the RMSEA and RMR values were smaller than 0.08 and 0.1 , respectively, verifying the model validity. Moreover, the GFI and AGFI were greater than 0.9 , demonstrating the verifiability of their factor structure and the acceptable model fit (Table 4). Given the relative fit of the confirmatory factor model and the significant item-scale relationship, the results of the exploratory factor model were supported by confirmatory patterns, and the construct validity of the scale was verified (Fig. 1).

\section{Discriminant validity}

Maternal age had a significant inverse correlation with the overall maternal functioning score $(\mathrm{r}=-0.18, p<$ 0.001). In addition, maternal age had a significant 
Table 3 Factor loadings of the Barkin Index of Maternal Functioning (BIMF) $(n=530)$

\begin{tabular}{lll}
\hline Items & Factor 1 & Factor 2 \\
\hline BMFI1 & 0.525 & \\
BMFI2 & 0.585 & \\
BMFI3 & 0.454 & \\
BMFI4 & 0.750 & \\
BMFI5 & 0.688 & 0.936 \\
BMFI6 & & 0.936 \\
BMFI7 & & 0.413 \\
BMFI8 & & \\
BMFI9 & & \\
BMFI10 & 0.610 & \\
BMFI11 & 0.762 & \\
BMFI12 & 0.504 & \\
BMFI13 & 0.739 & \\
BMFI14 & 0.537 & \\
BMFI15 & 0.775 & \\
BMFI16 & & \\
BMFI17 & & \\
BMFI18 & & \\
BMFI19 & & \\
BMFI20 & 0.73 .2 & \\
\% Variance Explained & 0.329 & \\
\hline
\end{tabular}

inverse correlation with the sub-scales of maternal needs $(\mathrm{r}=-0.19, p<0.001)$ and maternal competency $(\mathrm{r}=-$ $0.11, p=0.008)$. Gestational age did not have a significant correlation with the overall maternal functioning score and its sub-scales $(P>0.05)$. A significant difference in the maternal needs $(p<0.001)$ and the overall maternal functioning score $(p<0.001)$ was observed

Table 4 Confirmatory factor analyses fit Index of the Barkin Index of Maternal Functioning (BIMF) $(n=530)$

\begin{tabular}{ll}
\hline Fit Indices & Fit \\
\hline$x^{2} / d f$ & 4.90 \\
RMSEA (90\%Cl) & 0.07 (0.073 to 0.08) \\
GFI & 0.94 \\
AGFI & 0.90 \\
$\mathrm{NFI}$ & 0.92 \\
$\mathrm{IFI}$ & 0.95 \\
$\mathrm{TLI}$ & 0.93 \\
$\mathrm{CFI}$ & 0.95
\end{tabular}

$x^{2} / d f$ : Normed chi-square; RMSEA Root Mean Square Error of Approximation, GFI Goodness of Fit Index, AGFI Adjusted Goodness of Fit Index, NFI Normed Fit Index, IFI Incremental Fit Index, TLI Tucker- Lewis Index, CFI Comparative Fit Index between mothers who had been supported and those who had not received any support (Table 5).

\section{Reliability}

Cronbach's alpha coefficient of the questionnaire items was calculated from 0.78 (maternal competency) to 0.86 (maternal needs). Cronbach's alpha coefficient of the overall 20 -item version was calculated as 0.88 , indicating the acceptable internal consistency of the questionnaire. In the test-retest method, the ICC (95\% Confidence Interval) of the total questionnaire, and the constructs of maternal needs and maternal competency were calculated as 0.85 (0.64 to 0.94$), 0.89$ (0.59 to 0.93$)$, and 0.88 (0.71 to 0.95$)$, respectively (Table 6$)$.

\section{Discussion}

The present study was conducted in order to assess the psychometric properties of the BIMF in a sample of Iranian mothers. The results showed that the Persian version of the BIMF is a valid and reliable instrument for assessing maternal functioning among Iranian mothers. The face, content and construct validity, internal consistency, and test-retest reliability of the instrument were all confirmed.

Based on exploratory analysis, Items 15 and 18 were removed from the Persian version on account of their low factor loadings. In addition, Item 16 was not included under any of the factors and was therefore removed. Items 16 and 18 had also been removed in the psychometric assessment of the Turkish version of the instrument due to their low factor loadings. Moreover, Item 15 was reported to have been inappropriately labeled under one of the factors. The findings of the psychometric assessment of the Turkish version are consistent with the Persian version [27]. The culture of Iran is similar to Turkey's and thus, the consistency is justified.

Barkin et al. (2014) [6] evaluated the psychometric properties of the original version, in which they also excluded Items 16 and 18 from their analysis due to inadequate factor loadings. Barkin et al. eventually introduced an 18-item version of the instrument as well, though they recommend the 20-item version as it includes questions on anxiety and worry, which are important considerations in the postpartum period. Items 16 and 18 are the only items that address anxiety, worry, and mother-child interaction and they are valuable for practical purposes. For example, if an organization assessed maternal functioning using a shortened version of the BIMF, but did not screen for depression simultaneously, there would be no gauge of maternal anxiety, which is highly prevalent in the postpartum period [28]. Therefore, the Persian version of the BIMF can be 


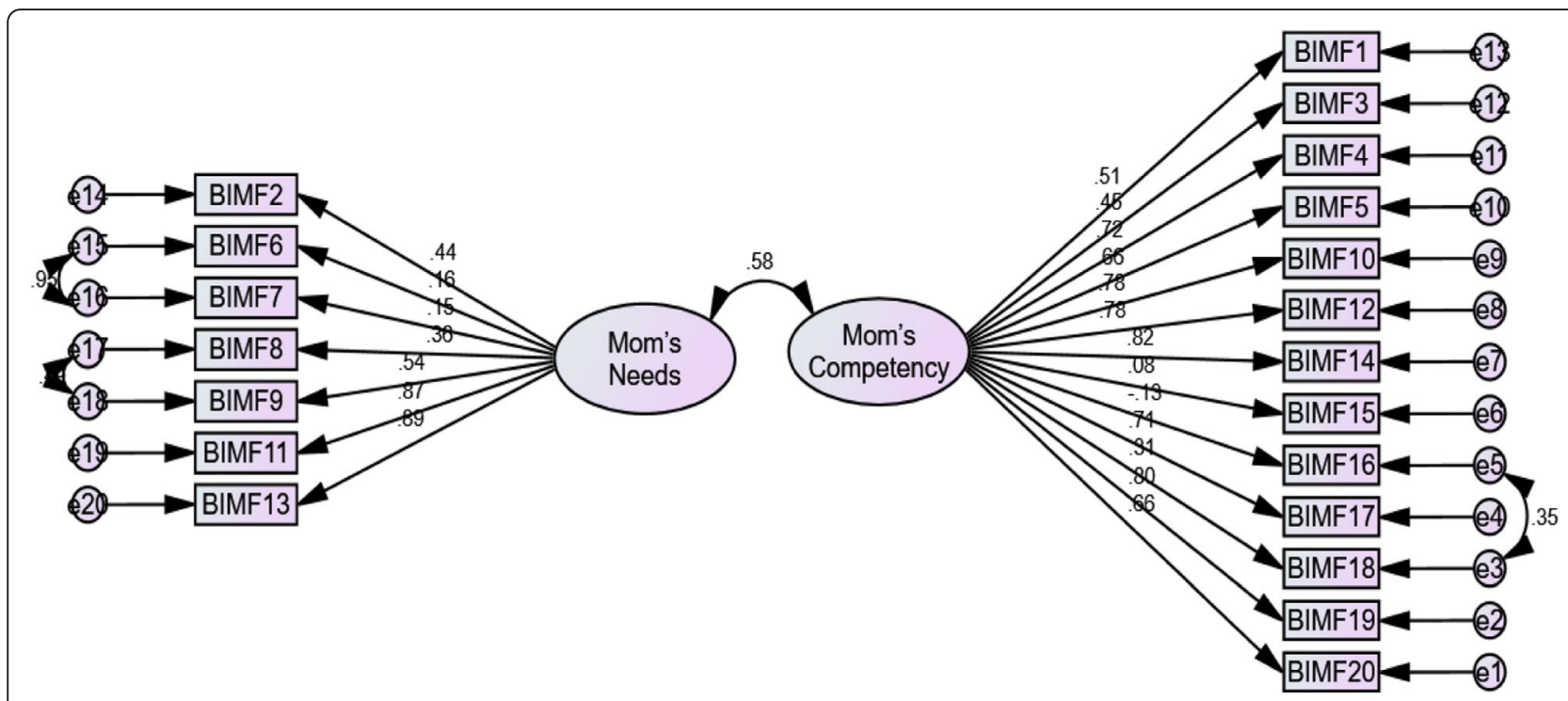

Fig. 1 CFA factor loading for Barkin Index of Maternal Functioning (BIMF)

administered in its 17 -item version or in its original 20item version.

Two factors were extracted from the Persian version: maternal needs (items 6, 7, and 8) and maternal competency (items 1, 2, 3, 4, 5, 9, 10, 11, 12, 13, 14, 17, 19, and 20). The number of factors extracted from the Turkish version is not consistent with those from the Persian version [27]. In the Turkish version, the five factors are named "self-care", "child care", "maternal psychology", "maternal attachment", "maternal management", "social support", and "compatibility with the maternal role" [27]. However, the number of factors extracted from this study is consistent with those from the Barkin et al.'s (2014) study. In their study of the psychometric properties of the instrument, they also discovered only two latent factors "maternal needs" and "maternal competency" [6].

The internal consistency was 0.88 for the Persian version and ranged from 0.78 (maternal competency) to 0.86 (maternal needs) for its dimensions. In the original (English) version [6], the Cronbach's alpha coefficients

Table 5 Discriminante validity for the Barkin Index of Maternal Functioning (BIMF) and it's sub scales $(n=530)$

\begin{tabular}{llll}
\hline Variables & Mom's needs & Mom's competency & Total \\
\hline Mother's age & & & \\
$r$ & -0.19 & -0.11 & -0.18 \\
P-value* & $<0.001$ & 0.008 & $<0.001$ \\
Receiving support & & \\
Yes $^{\&}$ & $34.3(6.6)$ & $63.5(6.9)$ & $97.9(11.8)$ \\
No $^{\&}$ & $28.1(6.5)$ & $62.3(7.0)$ & $90.5(11.1)$ \\
P-value $^{* *}$ & $<0.001$ & 0.054 & $<0.001$ \\
\hline
\end{tabular}

${ }^{*}$ Pearson correlation ${ }^{\&}$ Mean (SD) **Independent T-test of the 20-item version, and the dimensions of maternal needs and maternal competency were 0.87, 0.77, and 0.88 , respectively - in line with the results of the internal consistency of the Persian version. However, the Cronbach's alpha of the Turkish version [27] was reported as 0.73, which was lower than that of the Persian version. Such a distinction can be attributed to the greater number of items in the factors of the Persian and English versions, as items are scattered around five factors in the Turkish version instead of two. The ICC of "maternal needs" and "maternal competency" in the Persian version was 0.89 and 0.88 , respectively, which was consistent with the English version (0.80 and 0.88).

In this study, the total BIMF score and its sub-scale's scores had a significant inverse correlation with maternal age. In other words, older mothers had a weaker maternal functioning than younger mothers. Barkin et al. [19] also found a significant inverse correlation between maternal age and maternal competency, which is relatively consistent with the findings of this study. In addition, a significant difference in the maternal needs and the overall maternal functioning score was observed between mothers who had been supported and those who had not received any support. In a study by Giallo et al. [10] women who had been supported by home visits during the postpartum period were more capable of adapting to anxiety and depression and exhibited more favorable maternal behavior than the control group. Another study also reported that women who are supported during the postpartum period through pre-natal and postnatal training programs, home visits and telephone counseling, feel more self-sufficient and competent in self-care and neonatal care and have a better maternal functioning [29]. 
Table 6 Mean (SD), Cronbach's alpha, and ICC for the Barkin Index of Maternal Functioning (BIMF) $(n=530)$

\begin{tabular}{llllll}
\hline BMFI & Mean $\left(S D^{\text {a }}\right)$ & Possible range & Obtainable range & Cronbach's alpha $^{\text {ICC (95\% Cl) }}$ \\
\hline Mom's needs & $32.0(7.2)$ & $0-42$ & $7-42$ & 0.86 & $0.89(0.59$ to 0.93$)$ \\
Mom's competency & $63.1(6.9)$ & $0-78$ & $27-78$ & 0.78 & $0.88(0.71$ to 0.95) \\
Total & $95.2(12.1)$ & $0-120$ & $39-119$ & 0.88 & 0.85 (0.64 to 0.94)
\end{tabular}

${ }^{\mathrm{a}} \mathrm{SD}$ Standard Deviation ${ }^{\mathrm{b}} \mathrm{ICC}(95 \% \mathrm{Cl})$ Intarclass Correlation Coefficient (95\% Confidence Interval)

\section{Strengths and limitations}

The large sample size and the use of random cluster sampling are strengths and enhance the generalizability of this study to Iranian mothers outside of the study population. Non-inclusion of mothers with cesarean and multiparous delivery is a limitation of this study, as the psychometric assessment of the instrument is not applicable to all of these groups. However, the fact that our results are largely in agreement with the Barkin et al. (2014) psychometric analysis -which did not exclude women who had a cesarean section- is reassuring as to the generalizability. Failure to account for mothers affected by intimate partner violence -a proven stressorcould be considered an omission. However, none of the related studies thus far have included intimate partner violence as an explanatory variable.

\section{Conclusion}

The findings of this study revealed that the Persian version of the BIMF is a valid and reliable instrument for assessing postpartum maternal functioning in Iranian women. This instrument can be used by healthcare providers such as doctors, midwives, and nurses to screen and examine the compatibility of mothers with the maternal role in the postpartum period.

\section{Abbreviations \\ AGFI: Adjusted Goodness of Fit Index; BIMF: Barkin Index of Maternal Functioning; CFI: Comparative Fit Index; CVl: Content validity index; CVR: Content Validity Ratio; GFI: Goodness of Fit Index; ICC: Intra-class Correlation Coefficient; IFI: Incremental Fit Index; IFSAC: Inventory of Functional Status After Childbirth; KMO: Kaiser-Meyer-Olkin; NFI: Normed Fit Index; RMR: Root Mean Square Residual; RMSEA: Root Mean Square Error of Approximation; RMSEA: Root Mean Square Error of Approximation; TLI: Tucker- Lewis Index}

\section{Acknowledgements}

The authors appreciate the cooperation of all employees of healthcare centers in Tabriz as well as the mothers who participated in this study.

\section{Authors' contributions}

MM, JLB, MAJ and SGH contributed to the design of the study. FK and SGH contributed to the implementation. MAJ and MM contributed to the analysis plan. MM, SGH, FK and JLB has written the first draft of this manuscript and all authors have critically read the text and contributed with inputs and revisions, and all authors read and approved the final manuscript.

\section{Funding}

This study was funded by the Tabriz University of Medical Sciences, Tabriz, Iran (code: IR.TBZMED.REC.1397.147). The funding body had no role in the design of the study, the collection, analysis, and interpretation of data and in writing the manuscript.
Availability of data and materials

Not applicable.

Ethics approval and consent to participate

Ethical approval for the study was obtained from the Research Ethics Committee of the Tabriz University of Medical Sciences (Ethical code: IR.TBZMED.REC.1397.147). All participants provided written informed consent.

\section{Consent for publication}

Not applicable.

\section{Competing interests}

The authors declare that they have no competing interests.

\section{Author details}

${ }^{1}$ Social determinants of Health Research Center, Tabriz University of Medical Sciences, Tabriz, Iran. ${ }^{2}$ Department of Community Medicine, Mercer University School of Medicine, Macon, GA, USA. ${ }^{3}$ Department of Statistics and Epidemiology, Tabriz University of Medical Sciences, Tabriz, Iran. ${ }^{4}$ Road Traffic Injury Research Center, Tabriz University of Medical Sciences, Tabriz, Iran. ${ }^{5}$ MSc Student of Midwifery, Students' Research Committee, Tabriz University of Medical Sciences, Tabriz, Iran. ${ }^{6}$ Department of Midwifery, Faculty of Nursing and Midwifery, Tabriz University of Medical Sciences, Tabriz, Iran.

Received: 3 September 2019 Accepted: 29 November 2019

Published online: 21 December 2019

\section{References}

1. Mercer RT. Nursing support of the process of becoming a mother. J Obstet Gynecol Neonatal NSurs. 2006;35(5):649-51.

2. Don BP, Chong A, Biehle SN, Gordon A, Mickelson KD. Anxiety across the transition to parenthood: change trajectories among low-risk parents. Anxiety Stress Coping. 2014;27(6):633-49.

3. Meleis Al. Transitions theory: middle range and situation specific theories in nursing research and practice: springer publishing company; 2010.

4. Negron R, Martin A, Almog M, Balbierz A, Howell EA. Social support during the postpartum period: mothers' views on needs, expectations, and mobilization of support. Matern Child Health J. 2013:17(4):616-23.

5. Nelson AM. Transition to motherhood. J Obstet Gynecol Neonatal Nurs. 2003:32(4):465-77

6. Barkin $J \mathrm{~L}$, Wisner $\mathrm{KL}$, Wisniewski SR. The psychometric properties of the Barkin index of maternal functioning. J Obstet Gynecol Neonatal Nurs. 2014; 43(6):792-802

7. Smith-Hanrahan C, Deblois D. Postpartum early discharge: impact on maternal fatigue and functional ability. Clin Nurs Res. 1995;4(1):50-66.

8. McVeigh CA. Anxiety and functional status after childbirth. Aust Coll Midwives Inc J. 2000;13(1):14-8.

9. Ngai FW, Wai-Chi Chan S, Ip WY. Predictors and correlates of maternal role competence and satisfaction. Nurs Res. 2010;59(3):185-93.

10. Giallo R, Cooklin A, Dunning M, Seymour M. The efficacy of an intervention for the management of postpartum fatigue. J Obstet Gynecol Neonatal Nurs. 2014;43(5):598-613

11. Barkin $J$, Wisner $\mathrm{KL}$. The role of maternal self-care in new motherhood. Midwifery. 2013;29(9):1050-5.

12. Barkin JL, Wisner KL, Bromberger JT, Beach SR, Wisniewski SR. Assessment of functioning in new mothers. J Women's Health (Larchmt). 2010;19(8):1493-9.

13. Barkin JL, Beals L, Bridges CC, Ezeamama A, Serati M, Buoli M, ... \& Bloch JR. Maternal Functioning and Depression Scores Improve Significantly With Participation in Visiting Moms ${ }^{\circledR}$ Program. J Am Psychiatr Nurses Assoc. 2019; $1-10$. 
14. Fawcett J, Tulman L, Myers ST. Development of the inventory of functional status after childbirth. J Nurse Midwifery. 1988;33(6):252-60.

15. McVeigh C. Functional status after childbirth in an Australian sample. J Obstet Gynecol Neonatal Nurs. 1998;27(4):402-9.

16. Aktan NM. Functional status after childbirth: a review of the literature. Clin Nurs Res. 2007;16(3):195-211.

17. McVeigh C, Chaboyer W. Reliability and validity of the inventory of functional status after childbirth when used in an Australian population. Nurs Health Sci. 2002;4(3):107-12.

18. Barkin JL, Wisner KL, Bromberger JT, Beach SR, Terry MA, Wisniewski SR. Development of the Barkin index of maternal functioning. J Women's Health (Larchmt). 2010;19(12):2239-46.

19. Barkin JL, Willis GB, Hawkins KC, Stanfill-Thomas T, Beals L, Bloch JR. Semantic assessment of the Barkin index of maternal functioning in a medically underserved obstetric population. Perspect Psychiatr Care. 2017; 53(2):95-103.

20. Barkin JL, Bloch JR, Hawkins KC, Thomas TS. Barriers to optimal social support in the postpartum period. J Obstet Gynecol Neonatal Nurs. 2014; 43(4):445-54.

21. Nunnally JC, Bernstein IH. Psychometric Theory. New York: Mc Graw-hill; 1994.

22. Seyf AA. Measurement, test and educational evaluation. 7 ed. Tehran: Douran; 2016

23. Hajizadeh E, Asghari M. Statistical methods and analyses in health and biosciences a research methodological approach. Tehran: Jahade Daneshgahi Publications. 2011:395-450.

24. Lawshe CH. A quantitaitive approach to content validity. Pers Psychol. 1975; 28:563-75.

25. Tinsley HE, Brown SD. Handbook of applied multivariate statistics and mathematical modeling: academic press; 2000

26. Bentler PM, Bonett DG. Significance tests and goodness of fit in the analysis of covariance structures. Psychol Bull. 1980;88(3):588.

27. Aydin R, Kukulu K. Adaptation of the Barkin scale of maternal functioning and examination of the psychometric properties. Health Care Women Int. 2018:39(1):50-64.

28. Fairbrother $\mathrm{N}$, Janssen $\mathrm{P}$, Antony MM, Tucker E, Young AH. Perinatal anxiety disorder prevalence and incidence. J Affect Disord. 2016;200:148-55.

29. Aydın R, Barkin JL, Kukulu K. Attempts to strengthen maternal functioning in the postpartum period: a literature review. J Hum Sci. 2016;13(3):5751-9.

\section{Publisher's Note}

Springer Nature remains neutral with regard to jurisdictional claims in published maps and institutional affiliations.

Ready to submit your research? Choose BMC and benefit from:

- fast, convenient online submission

- thorough peer review by experienced researchers in your field

- rapid publication on acceptance

- support for research data, including large and complex data types

- gold Open Access which fosters wider collaboration and increased citations

- maximum visibility for your research: over $100 \mathrm{M}$ website views per year

At $\mathrm{BMC}$, research is always in progress.

Learn more biomedcentral.com/submissions 